\title{
Rietveld and pair-distribution function analysis of nanogranular mesocrystalline shells of hyaline foraminifers
}

\author{
A.I. Arns ${ }^{1,2,3}$, R. Schiebel ${ }^{1}$, D. Evans ${ }^{2}$, L. Fink ${ }^{3}$, E. Alig ${ }^{3}$, M.U. Schmidt ${ }^{3}$, A. Jantschke ${ }^{4}$, J. Linckens ${ }^{2}$, G.H. Haug ${ }^{5}$ \\ ${ }^{1}$ Department of Climate Geochemistry, Max Planck Institute for Chemistry, Hahn-Meitner-Weg 1, 55128 Mainz, Germany, ${ }^{2}$ Institute \\ of Geosciences, Goethe University Frankfurt, Altenhöferallee 1, 60438 Frankfurt a.M., Germany, ${ }^{3}$ Institute of Inorganic and \\ Analytical Chemistry, Goethe University Frankfurt, Max-von-Laue-Str. 7, 60438 Frankfurt a.M., Germany ${ }^{4}$ Institute of Geosciences, \\ Johannes Gutenberg University Mainz, Johann-Joachim-Becher-Weg 21, 55128 Mainz, Germany, ${ }^{5}$ Department of Earth Sciences, \\ ETH Zurich, Sonneggstrasse 5, 8092 Zurich, Switzerland \\ anthea.arns@mpic.de
}

Foraminifers are unicellular marine organisms which form a carbonate shell ('test') that is made of consecutively mineralised chambers. Due to the high abundance and good preservation of these tests in ocean sediments, foraminifers are a key component of the global carbon cycle and provide an outstanding archive for paleoclimate reconstruction. The exact mechanism of biomineralisation in foraminifers is not known, which results in uncertainties both in their reaction to climate change and ocean acidification, as well as for the interpretation of element and isotope proxies for past climate conditions measured on fossil tests.

To address this, we investigated the crystal structure and structural hierarchy of the tests of planktic and benthic hyaline foraminifer species from different suborders, including both modern and fossil specimens dating back to approx. 5 Ma. A multi-technique approach was taken, including Laser Ablation Inductively Coupled Plasma Mass Spectrometry, Electron Backscatter Diffraction (EBSD), Scanning Electron Microscopy (SEM), as well as Rietveld refinement and Pair Distribution Function (PDF) analysis based on laboratory X-ray diffraction measurements. The investigated tests show no resolvable crystallographic difference between modern and fossil specimens. Crystalline metastable phases were not observed in any of the specimens. In modern samples, a slight elevation of the amorphous diffraction background is present, which could be attributed either to amorphous carbonates or organic residues. The unit cell parameters of biogenic Mg-calcites of the foraminifer shells were determined by Rietveld refinement to be close to inorganic Mgcalcite of the respective Mg-content. Crystallite dimensions for the investigated hyaline foraminifer tests range between 40-150 nm, as determined by Rietveld refinement, and at least 30-40 nm by PDF analysis. The small dimension of coherently diffracting crystallite domains is supported by a nanogranular surface morphology of mechanically fractured chamber walls in SEM, which exhibits irregularly formed units 100-300 nm in size. EBSD analysis demonstrates the presence of uniformly scattering regions in the test of the planktic species $G$. ruber with a diameter of several micrometres and the crystallographic $c$-axis of the grains oriented perpendicular to the chamber surface, which is a feature observed in several other hyaline foraminifer species [1]. This indicates that the tests of the investigated species are built of micrometre-sized mesocrystals made of aligned nanometre-sized entities. We hypothesise that a coalescence of the nanocrystallites is prevented by the presence of an amorphous margin around the entities, possibly organic- and/or impurity-rich, which is supported by the observation of amorphous matter at grain boundaries in a different hyaline foraminifer species

[2].

The presence of nanogranular mesocrystals in hyaline foraminifer test calcites together with the distinct orientation of mesocrystal grains indicates a biochemically controlled biomineralisation mechanism, which follows a non-classical crystallisation pathway [2,3]. This supports the notion that foraminiferal biomineralisation involves the formation of a metastable precursor phase such as amorphous $\mathrm{CaCO} 3$, possibly in interaction with an organic matrix, which is followed by directed crystallisation. This could suggest an involvement of organic matter in hyaline foraminifera biomineralisation not only as a template for mineralisation [4], but also as a surface and matrix for nucleation.

Hence, as a next step to resolve test biomineralisation mechanisms in foraminifers and to improve our understanding of the relation of proxies and test structure to environmental parameters, the composition and function of the organic material present at the site of mineralisation needs to be better understood, and the influence of organics on the nucleation and crystallisation of carbonates should be further studied in experimental models.

[1] Read, E. (2019). PhD thesis, University of Cambridge, United Kingdom

[2] Jacob, D. E., Wirth, R., Agbaje, O. B. A., Branson, O. \& Eggins, S. M. (2017). Nature Communications. 8, 1-8.

[3] Wolf, S. E., Böhm, C. F., Harris, J., Demmert, B., Jacob, D. E., Mondeshki, M., Ruiz-Agudo, E. \& Rodríguez-Navarro, C. (2016). Journal of Structural Biology. 196, 244-259.

[4] Towe, K. \& Cifelli, R. (1967). Journal of Paleontology. 41, 742-762.

Keywords: Biomineralization; Foraminifera; Mesocrystal; Nonclassical Crystallization

Acta Cryst. (2021), A77, C1069 